\title{
Zu axialsymmetrischen stationären Lösungen der Einsteinschen Feldgleichungen für das Vakuum
}

\author{
D. Kramer und G. Neggebauer \\ Theoretisch-Physikalisches Institut der Friedrich-Schiller-Universität Jena \\ Eingegangen am 8. April 1968
}

\begin{abstract}
For axialsymmetric stationary vacuum fields Einstein equations reduce to a system, derivable from a simple Lagrangian. An investigation of its forminvariance leads to a method to construct from known solutions generalized solutions with one additional parameter.

The method is applied to Weyl's class and to Kerr metric.

Für axialsymmetrische stationäre Vakuumfelder reduzieren sich die Einsteinschen Gleichungen auf ein System, das aus einer einfachen Lagrangefunktion herleitbar ist. Eine Untersuchung ihrer Forminvarianz führt zu einer Methode, die es erlaubt, aus bekannten Lösungen verallgemeinerte Lösungen mit einem zusätzlichen Parameter zu konstruieren.

Das Verfahren wird auf die Weylsche Klasse und auf die Kerr-Metrik angewandt.
\end{abstract}

\section{Einleitung}

Uns interessieren hier diejenigen Lösungen der Einsteinschen Feldgleichungen für das Vakuum, bei denen eine Abelsche Bewegungsgruppe $G_{2}$ vorliegt. Der zeitartige Killingvektor $\xi^{\mu}$ drückt die Stationarität aus, der raumartige Killingvektor $\eta^{\mu}$, dessen Trajektorien konzentrische Kreise um die Symmetrieachse seien, beschreibt die Axialsymmetrie. Beide Killingvektorfelder sind im allgemeinen nicht hyperflächennormal. Unter diesen Voraussetzungen allein lassen sich die Feldgleichungen noch nicht integrieren, die statischen axialsymmetrischen Lösungen (Weylsche Klasse) sind jedoch vollständig bekannt. Wir geben ein Verfahren an, wie man aus bekannten Lösungen (mit $G_{2}$ ) andere mit zusätzlichem Parameter gewinnen kann.

\section{Feldgleichungen}

Wenn die Feldgleichungen $R_{\mu \nu}=0$ außerhalb einer inselförmigen Materieverteilung auch auf der Achse erfüllt sein sollen, läßt sich nach [1] das Linienelement eines Raumes mit den Killingvektoren $\xi^{\mu}$ und $\eta^{\mu}$ ohne zusätzliche Annahmen auf folgende Form bringen:

$$
\begin{aligned}
d s^{2} & =e^{-2} U\left(\gamma_{A B} d x^{A} d x^{B}+W^{2} d \varphi^{2}\right)-e^{2} U(d t+a d \varphi)^{2} \\
\xi^{\mu} & =\delta_{0}^{\mu}, \quad \eta^{\mu}=\delta_{3}^{\mu} \quad \varphi \equiv x^{3}, \quad t \equiv x^{0}, \quad A=1,2 .
\end{aligned}
$$


Dabei hängen die metrischen Funktionen $\gamma_{A B}, W, U, a$ nur von den beiden Variablen $x^{A}$ ab. Im Zweierraum $V_{2}$ mit der Metrik $\gamma_{A B}$ können noch beliebige Koordinatentransformationen

$$
x^{A^{\prime}}=x^{A^{\prime}}\left(x^{B}\right)
$$

ausgeführt werden.

Setzt man in der Metrik (1) voraus, daß einer der beiden Killingvektoren hyperflächennormal ist, so ergibt sich unmittelbar die Existenz eines zweiten hyperflächennormalen Killingvektors. Damit gilt

Satz I. Die Bondi-Metrik [2] enthält keine eigentlich-stationären, sondern nur statische Lösungen mit $G_{2}$.

Stationäre axialsymmetrische Massenströme in den Ebenen $\varphi=$ const. müßten sich also, wenn sie überhaupt existieren können, an eine statische Weylsche Vakuumlösung anschließen lassen. Stationäre Rotationen um die Symmetrieachse sind jedoch mit den Feldgleichungen vereinbar und in der von SACHS [3] angegebenen Verallgemeinerung der Bondi-Metrik enthalten.

Satz I läßt sich auch ohne das Resultat von [1] zeigen [4].

Anstelle von $a \equiv e^{-2} U g_{03}$ führen wir jetzt gemäß1

$$
b,_{A}=\frac{e^{4} U}{W} \varepsilon_{A C} a^{\prime C}
$$

eine neue Funktion $b$ ein. Damit folgt aus den Feldgleichungen $R_{\mu \nu}=0$ :

$$
\begin{gathered}
W,,_{A} ; A=0, \\
\frac{1}{W}\left(W U,_{A}\right) ; A=-\frac{1}{2} e^{-4 U} b,_{A} b^{\prime A}, \\
\frac{1}{W}\left(W b,_{A}\right) ; A=4 U,_{A} b^{\prime A} .
\end{gathered}
$$

Die metrischen Operationen beziehen sich auf $\gamma_{A B}$.

(6) ist die Integrabilitätsbedingung zu (3); die ursprünglich für $a$ geltende Differentialgleichung ist durch den Ansatz (3) identisch erfüllt. Ein Konformfaktor in der Metrik $\gamma_{A B}$ geht in die Gln. $(4-6)$ nicht ein. Die restlichen Feldgleichungen liefern dann gerade die Bestimmungsgleichung für diesen Konformfaktor.

Mit Hilfe von (2) lassen sich in $V_{2}$ immer isotrope Koordinaten einführen:

$$
\gamma_{A B}=e^{2 k} \delta_{A B}
$$

Da $W$ in diesen Koordinaten Potentialfunktion ist, können wir durch eine Transformation

$$
z^{\prime}=w(z), \quad z \equiv x^{1}+i x^{2}, \quad w(z) \text { analytisch }
$$

\footnotetext{
${ }^{1} \varepsilon_{A B}=$ Levi-Civita-Pseudotensor in $V_{2}\left(\right.$ Metrik $\left.\gamma_{A B}\right)$.
} 
stets $W=x^{1}$ erreichen. Die isotrope Form von $\gamma_{A B}$ wird dabei nicht zerstört. Damit ist (4) identisch befriedigt, und das Problem reduziert sich auf das gekoppelte System elliptischer Differentialgleichungen

$$
\begin{aligned}
\frac{1}{x^{1}}\left(x^{1} U,_{A}\right),_{A} & =-\frac{1}{2} e^{-4 U} b,_{A} b,_{A} \\
\left.\frac{1}{x^{1}}\left(x^{1} b,_{A}\right)\right)_{A} & =4 U,_{A} b,_{A} .
\end{aligned}
$$

$\mathrm{Zu}$ jeder Lösung $U, b$ dieses Systems ist dann das zugehörige $k$ durch ein Linienintegral aus

$$
k_{z}=\frac{x^{1}}{2}\left[\left(U,_{z}\right)^{2}+\frac{1}{4} e^{-4 U}\left(b,,_{z}\right)^{2}\right] \quad\left(z \equiv x^{1}+i x^{2}\right)
$$

bestimmbar, wobei die Integrabilitätsbedingung wegen (9) automatisch erfüllt ist.

Die Weylsche Klasse ist als Spezialfall $(b=0)$ enthalten.

Auf das System (9) wird man auch bei der Behandlung anderer Probleme geführt. Zum Beispiel läßt sich jeder axialsymmetrischen stationären Lösung von $R_{\mu \nu}=0$ eine axialsymmetrische statische Lösung mit elektrostatischem Feld zuordnen, indem man in (1) $a \equiv 0$ setzt und die Substitutionen

$$
\begin{aligned}
2 U & \rightarrow U, \\
b & \rightarrow \sqrt{\frac{-x}{2}} \chi
\end{aligned}
$$

$(\varkappa=$ Einsteinsche Gravitationskonstante, $\chi=$ elektrostatisches Potential) sowie Parameteränderungen $(l \rightarrow i l)$ vornimmt. Die Lösung, die in diesem Sinne das Analogon zur Kerr-Metrik [5] darstellt, wurde in [6] angegeben und diskutiert. Umgekehrt entspricht beispielsweise der Reissner-Nordström-Lösung eine stationäre Lösung, bei der allerdings die Bedingung

$$
a \sim \frac{\sin ^{2} \vartheta}{r}+\left(\text { höhere Potenzen in } \sin \vartheta \text { und } r^{-1}\right)
$$

nicht erfüllt ist $(a \sim \cos \vartheta)$. (12) wird durch die linearisierte Theorie nahegelegt [7] und ermöglicht

(1) Übergang des Linienelementes in die Metrik des flachen Raumes für große räumliche Abstände $r$,

(2) Mikroeuklidizität auf der Symmetrieachse [8]. (Das Verhältnis von Umfang zu Radius eines Kreises um die Achse ergibt im Limes kleiner Radien gerade $2 \pi$.)

Das Gleichungssystem (9) bleibt gegenüber der Ersetzung

$$
\begin{aligned}
e^{2 U} & =x^{1} e^{-2 \tilde{U}} \\
b,,_{A} & =i x^{1} e^{-4 \tilde{U}} \varepsilon_{A B} \tilde{b},_{B}=-i \tilde{a},,_{A}
\end{aligned}
$$


forminvariant. (Der Faktor $i \equiv \sqrt{-1}$ kann durch Parameteränderungen ausgeglichen werden.) Aus einer Lösung $\tilde{U}, \tilde{b}$ erhält man so eine neue Lösung $U, b$.

\section{Invarianzen der Lagrangefunktion}

Das System (9) ist aus der Lagrangefunktion

$$
L=x^{1}\left(U,_{A} U,_{A}+\frac{1}{4} e^{-4 U} b,_{A} b,,_{A}\right)
$$

herleitbar $\left(\frac{\delta L}{\delta U}=\frac{\delta L}{\delta b}=0\right)$. Beim formalen Übergang von Zylinderkoordinaten zu dreidimensionalen kartesischen Koordinaten ergibt sich

$$
\begin{gathered}
L=U,_{i} U,_{i}+\frac{1}{4} e^{-4 U} b_{i}, b_{i}, \quad i=1,2,3 \\
{\left[\Delta U \equiv U_{i},,_{i}=\frac{1}{x^{1}}\left(x^{1} U,_{A}\right),{ }_{A}\right] .} \\
f \equiv e^{2} U+i b
\end{gathered}
$$

erhält man schließlich aus $(15)^{2}$

$$
L=\frac{f_{i,} f_{i}^{*}}{\left(f+f^{*}\right)^{2}} \quad\left(f^{*} \equiv e^{2} U-i b\right) .
$$

Wir untersuchen die Forminvarianz von $L$ bei Transformationen

$$
U=U(\widetilde{U}, \tilde{b}), \quad b=b(\widetilde{U}, \tilde{b}) .
$$

Eine Differentialform $e^{2 k} d z d z^{*}$ bleibt nur bei analytischen Transformationen (8) forminvariant; (17) hat eine entsprechende Struktur, das heißt

und es ist noch die Gleichung ${ }^{3}$

$$
f=f(\tilde{f}),
$$

$$
\frac{f^{\prime} f^{* \prime}}{\left(f+f^{*}\right)^{2}}=\frac{1}{\left(f+f^{*}\right)^{2}}
$$

auszuwerten. Wir formulieren das Ergebnis in

Satz II. Die Lagrangefunktion bleibt nur gegenüber den Transformationen

forminvariant.

$$
f=\frac{\alpha}{f+i \beta}+i \gamma \quad(\alpha, \beta, \gamma \text { reelle Konstanten })
$$

Dieses Resultat läßt sich auch herleiten, indem man (15) als Differentialform

$$
, d s^{2 “}=d U^{2}+\frac{1}{4} e^{-4 U} d b^{2}
$$

${ }^{2}$ Diese Form wurde auch von F. J. ERnst; Phys. Rev. 168, 1415 (1968) angegeben (Zusatz bei der Korrektur).

$$
f^{\prime} \equiv \frac{d f}{d f}, \quad f^{* \prime} \equiv \frac{d f^{*}}{d f^{*}} \text {. }
$$


auffaßt. Die Krümmung dieses „Raumes“ ist konstant:

$$
K=-e^{2} U \frac{\partial^{2}}{\partial U^{2}}\left(e^{-2 U}\right)=-4 .
$$

Man kann daher diese Pseudosphäre in einen pseudoeuklidischen Raum einbetten:

$$
e^{2} U=\frac{1}{A+C}, \quad b=\frac{B}{A+C} .
$$

Wir werden so auf ein Variationsproblem mit

$$
L=A,_{i} A,{ }_{i}+B,{ }_{i} B,,_{i}-C,_{i} C,_{i}
$$

unter der Nebenbedingung

$$
A^{2}+B^{2}-C^{2}=-1
$$

geführt. Die Feldgleichungen erhalten die symmetrische Form

$$
\Delta A=\lambda A, \quad \Delta B=\lambda B, \quad \Delta C=\lambda C
$$

( $\lambda=L:$ Lagrangescher Multiplikator).

Bei dem entsprechenden mechanischen Problem (kräftefreie Bewegung eines Massenpunktes auf einer Pseudosphäre) ist $\lambda=$ const, und die Integration bereitet keinerlei Schwierigkeiten. Hier liegt aber eine Abhängigkeit von 2 unabhängigen Variablen vor.

Die Lorentzdrehungen im Raum der $A, B, C$ (3 Gruppenparameter) bilden die Invarianzgruppe von (24) und (25). Umrechnung auf die komplexe Variable $f$ ergibt das Resultat (21).

Eine ähnliche Untersuchung findet man in [9]. Dort wird allerdings die Funktion $b$ nicht eingeführt, und die reellen Lorentzdrehungen führen nicht zu neuen Lösungen, sie sind mit Koordinatentransformationen in der Raum-Zeit identisch.

Aus einer Lösung $f$ gewinnen wir gemäß (21) (Satz II) eine Lösung $f$, die zunächst 3 neue Parameter enthält, von denen aber $\gamma$ wegen (3) unwesentlich ist und $\alpha$ durch die Koordinatentransformation

$$
\varphi^{\prime}=\alpha^{-1 / 2} \varphi, \quad t^{\prime}=\alpha^{1 / 2} t
$$

zu 1 gemacht werden kann. Es bleibt nur ein wesentlicher Parameter. Das asymptotische Verhalten $f \rightarrow 1$ wird durch die Operation (21) nicht verändert, wenn wir

wählen.

$$
\alpha=\beta^{2}+1, \quad \gamma=\beta
$$

Bei Beschränkung auf statische Metriken $(\tilde{b}=0)$ und $\beta=0$ geht (21) in eine spezielle Form des Buchdahl-Theorems [10] über:

$$
U=-\tilde{U} \text {. }
$$




\section{Lösungen der Feldgleichungen}

An konkreten Beispielen soll gezeigt werden, daß (21) tatsächlich einen wesentlichen Parameter enthält und zu einer erweiterten Lösung führt.

1. Die Anwendung von Satz II auf die statischen Metriken ergibt die Lösungsklasse $U=U(b)$ [11]. Wir erhalten mit (28)

$$
e^{2} \bar{U}=\left(1+\beta^{2}\right) \cdot \frac{e^{2} \tilde{U}}{e^{4} \tilde{U}+\beta^{2}}, \quad b=\beta \cdot \frac{e^{4} \tilde{U}-1}{e^{4} \tilde{U}+\beta^{2}}, \quad \Delta \widetilde{U}=0
$$

und damit den Zusammenhang $(\beta \neq 0)$

$$
e^{4} U=1+\frac{\beta^{2}-1}{\beta} b-b^{2} .
$$

Für $\beta=1$ vereinfachen sich die Ausdrücke (30), (31) zu

$$
e^{-2 U}=\mathfrak{L} \mathfrak{j} \Psi \quad b=\mathfrak{T} \mathfrak{g} \Psi, \quad e^{4} U=1-b^{2}
$$

$(\Psi \equiv 2 \widetilde{U})$. Mit der Lösung der Potentialgleichung $\Delta \Psi=0$

$$
\Psi=\sum_{n=1}^{\infty} \frac{C_{n} P_{n}(\cos \vartheta)}{r^{n+1}}
$$

berechnet man die Funktion $a \mathbf{z u}$

$$
a=\sum_{n=1}^{\infty} \frac{C_{n}}{r^{n}}\left(\cos \vartheta P_{n}-P_{n-1}\right) \sim \frac{\sin ^{2} \vartheta}{r}(1+\ldots) .
$$

Für $n \geqq 1$ ist die Lösung also in Einklang mit der Bedingung (12), das Gravitationspotential enthält jedoch keinen Anteil $\sim 1 / r$ : Die Lösung beschreibt wahrscheinlich rotierende Massenmultipole. Die Erweiterung von (33) um einen Term $n=0$ zerstört die Eigenschaft (12).

Die Ersetzung (13) führt die Klasse $U(b)$ in eine andere Lösungsklasse über, die durch die Existenz eines hyperflächennormalen komplexen Killingvektors $\zeta^{\mu}$ gekennzeichnet ist:

$$
\begin{aligned}
\left(x^{1}\right)^{2} e^{-4 U} & =1+a^{2} \Leftrightarrow g_{00}=-g_{33}: \\
\zeta^{\mu} & =(0,0,1, i), \quad \zeta_{\mu} \sim \zeta_{\mu} .
\end{aligned}
$$

In diesem Fall lassen sich die Feldgleichungen natürlich auch direkt integrieren.

2. Wir wenden (21) auf die Kerr-Metrik an und erhalten nach einer Transformation $r \rightarrow r-\nu$ :

$$
f=1-2 \frac{m_{1}+i m_{2}}{Z} \cdot m_{1}, m_{2}, l: \text { reelle Konstanten }
$$

$\gamma_{A B} d x^{A} d x^{B}=\left(r^{2}-2 m_{1} r+l^{2} \cos ^{2} \vartheta-m_{2}^{2}\right)\left(d \vartheta^{2}+\frac{d r^{2}}{R^{2}}\right), W=R \sin \vartheta$

$$
R^{2} \equiv r^{2}-2 m_{1} r+l^{2}-m_{2}^{2}, \quad Z \equiv r+i\left(l \cos \vartheta+m_{2}\right)
$$


Die zugehörige Funktion $a$ berechnet man daraus zu

$$
a=\frac{2 l \sin ^{2} \vartheta\left(m_{1} r+m_{2}\right)+2 m_{2} \cos \vartheta R^{2}}{r^{2}-2 m_{1} r+l^{2} \cos ^{2} \vartheta-m_{2}^{2}}
$$

Zwischen den Parametern $m_{1}, m_{2}, v, \beta$ und $m$ (= Massenparameter in der Kerr-Metrik vor der Transformation (21)) bestehen die Relationen

$$
1+\beta^{2}=\frac{2 m}{v}, \quad m_{1}=m-\nu, \quad m_{2}= \pm v \beta .
$$

Für $\beta=m_{2}=0$ erhält man die Kerr-Lösung $\left(m_{1}=-m\right)$, für $l=0$ die NUT-Metrik [12]. Die mit Hilfe unseres Verfahrens gewonnene kombinierte Metrik (36) wurde in [13] angegeben. Die dort angewandte komplexe Fortsetzung sichert nicht von vornherein, daß man wieder eine Lösung der Feldgleichungen erhält.

Die Transformation

$$
\begin{aligned}
u & =t-2 m_{2} \varphi+F(r) \\
\varphi^{\prime} & =\varphi+G(r) \\
\frac{d F}{d r} & =-\frac{r^{2}+\left(l+m_{2}\right)^{2}}{R^{2}}, \quad \frac{d G}{d r}=-\frac{l}{R^{2}}
\end{aligned}
$$

stellt die Verbindung zu dem in [13] verwendeten Koordinatensystem her ${ }^{4}$.

Die Berechnung des Krümmungstensors zeigt, daß (36) für $m_{2} \neq 0$ nicht mit der Kerr-Lösung identisch ist.

Satz II führt also zu echten Erweiterungen; man gewinnt neue Lösungen.

Nach (11) erhalten wir die zu (36) analoge statische Lösung mit dem elektrostatischen Potential

$$
\chi=\sqrt{\frac{\overline{2}}{x}} \cdot \frac{2 m_{2} r-2 m_{1}\left(m_{2}+l \cos \vartheta\right)}{r^{2}-\left(l \cos \vartheta+m_{2}\right)^{2}} .
$$

Für alle Parameterwerte verhält sich diese Lösung auf der Symmetrieachse außerhalb eines endlichen Gebietes regulär; die Metrik hat das richtige Verhalten für $r \rightarrow \infty$. Die Quellstruktur haben wir noch nicht untersucht.

Herm Prof. Dr. E. Schmotzer und den Mitarbeitern der Arbeitsgruppe Relativitätstheorie danken wir für wertvolle Diskussionen.

4 In [13] sind bei der Angabe der Metrik Druckfehler aufgetreten, z. B. muß es in (3.2) richtig heißen: $g_{33}=-R^{2} \sin ^{2} \vartheta+A\left(A f^{2}-2 a \sin ^{2} \vartheta\right)$.

Parameteridentifizierungen: $m_{1} \equiv m_{0}, m_{2} \equiv b, l \equiv a$. 


\section{Literatur}

1. Papapetrou, A.: Ann. Inst. H. Poincaré, 83 (1966).

2. Bondi, H., M. G. J. van Der Burg, and A. W. K. Metzner: Proc. Roy. Soc. A 269, 21 (1962).

3. SaCHS, R. G.: Proc. Roy. Soc. A 270, 103 (1962).

4. Neugebauer, G.: Wiss. Zeitschr. der Univ. Jena (im Druck).

5. KerR, R. P.: Phys. Rev. Lett. 11, 237 (1963).

6. Bonnor, W. B.: Z. Phys. 186, 222 (1965).

7. Thirring, H.: Phys. Zeitschr. 19, 33 (1918).

8. Synge, J. L.: Relativity: The general theory. Amsterdam: North Holland Publ. Comp. 1960.

9. Matzner, R. A., and Ch. W. Misner: Phys. Rev. 154, 1229 (1967).

10. BuchdahL, H. A.: Phys. Rev. 115, 1325 (1959).

11. Papapetrou, A.: Ann. Phys. 12, 309 (1953).

12. Newman, E. T., L. Tamburino, and T. Untr: J. Math. Phys. 4, 915 (1963).

13. Demiax́ski, M., and E. T. Newman: Bull. l'Acad. Pol. Sci. 14, 653 (1966).

D. Kramer

G. NeUGeBaUER

Theoret.-Physik. Institut

der Universität

X 69 Jena 